\title{
Reduction of ventriculostomy-associated CSF infection with antibiotic-impregnated catheters in pediatric patients: a single-institution study
}

\author{
Shih-Shan Lang, MD, ${ }^{1,2}$ Bingqing Zhang, MPH, ${ }^{3}$ Hugues Yver, MD, ${ }^{4}$ Judy Palma, BS, ${ }^{2}$ \\ Matthew P. Kirschen, MD, PhD, ${ }^{3}$ Alexis A. Topjian, MD, MSCE, ${ }^{3}$ Benjamin Kennedy, MD, ${ }^{1,2}$ \\ Phillip B. Storm, MD, ${ }^{1,2}$ Gregory G. Heuer, MD, PhD, ${ }^{1,2}$ Janell L. Mensinger, PhD, ${ }^{5}$ and \\ Jimmy W. Huh, MD ${ }^{3}$
}

'Division of Neurosurgery, Department of Neurosurgery, Children's Hospital of Philadelphia, Perelman School of Medicine, University of Pennsylvania; ${ }^{2}$ Center for Data Driven Discovery in Biomedicine, Children's Hospital of Philadelphia; ${ }^{3}$ Department of Anesthesiology and Critical Care Medicine, Children's Hospital of Philadelphia, Perelman School of Medicine, University of Pennsylvania; ' ${ }^{2}$ Department of Pediatrics, Children's Hospital of Philadelphia, Perelman School of Medicine, University of Pennsylvania; and ${ }^{5}$ Department of Epidemiology and Biostatistics, Dornsife School of Public Health, Drexel University, Philadelphia, Pennsylvania

OBJECTIVE External ventricular drains (EVDs) are commonly used in the neurosurgical population. However, very few pediatric neurosurgery studies are available regarding EVD-associated infection rates with antibiotic-impregnated EVD catheters. The authors previously published a large pediatric cohort study analyzing nonantibiotic-impregnated EVD catheters and risk factors associated with infections. In this study, they aimed to analyze the EVD-associated infection rate after implementation of antibiotic-impregnated EVD catheters.

METHODS A retrospective observational cohort of pediatric patients (younger than 18 years of age) who underwent a burr hole for antibiotic-impregnated EVD placement and who were admitted to a quaternary care ICU between January 2011 and January 2019 were reviewed. The ventriculostomy-associated infection rate in patients with antibiotic-impregnated EVD catheters was compared to the authors' historical control of patients with nonantibiotic-impregnated EVD catheters.

RESULTS Two hundred twenty-nine patients with antibiotic-impregnated EVD catheters were identified. Neurological diagnostic categories included externalization of an existing shunt (externalized shunt) in 34 patients (14.9\%); brain tumor (tumor) in 77 patients (33.6\%); intracranial hemorrhage (ICH) in 27 patients (11.8\%); traumatic brain injury (TBI) in 6 patients (2.6\%); and 85 patients (37.1\%) were captured in an "other" category. Two of 229 patients $(0.9 \%$ of all patients) had CSF infections associated with EVD management, totaling an infection rate of 0.99 per 1000 catheter days. This is a significantly lower infection rate than was reported in the authors' previously published analysis of the use of nonantibiotic-impregnated EVD catheters $(0.9 \%$ vs $6 \%, p=0.00128)$.

CONCLUSIONS In their large pediatric cohort, the authors demonstrated a significant decline in ventriculostomy-associated CSF infection rate after implementation of antibiotic-impregnated EVD catheters at their institution.

https://thejns.org/doi/abs/10.3171/2019.5.FOCUS19279

KEYWORDS external ventricular drain; externalized shunt; CSF infection; pediatric; neurocritical care; ventriculitis; ventriculostomy

$\mathrm{P}$ EDIATRIC neurosurgical patients continue to represent a major group of patients who are treated in pediatric and neonatal ICUs. ${ }^{1,6}$ External ventricular drains (EVDs) are commonly used in infants and children to remove and sample CSF, manage hydrocephalus from a variety of disease processes, and monitor and treat increased intracranial pressure. ${ }^{3,12}$ However, placement of EVDs poses risks including infection. We previously described a large cohort of pediatric neurosurgical patients in a single quaternary pediatric ICU focusing on risk fac-

ABBREVIATIONS EVD = external ventricular drain; $\mathrm{ICH}=$ intracranial hemorrhage; $I Q R=$ interquartile range; $\mathrm{OR}=$ operating room; $\mathrm{TBI}=\mathrm{traumatic}$ brain injury. SUBMITTED April 1, 2019. ACCEPTED May 21, 2019.

INCLUDE WHEN CITING DOI: 10.3171/2019.5.FOCUS19279. 
tors associated with EVD infections in patients treated with nonantibiotic-impregnated catheters; the infection rate was 8.6 per 1000 catheter (EVD) days. ${ }^{15}$

In the adult neurosurgical population, there has been increasing use of antibiotic-impregnated EVD catheters. Some studies in adults have demonstrated that the use of antibiotic-impregnated EVD catheters has decreased the EVD-associated infection rate, whereas others have not. ${ }^{4,7-12,16,17}$ Studies of antibiotic-impregnated EVD catheters are lacking in the pediatric population. In one small study, the use of antibiotic-impregnated EVD catheters in the management of hydrocephalus in children with posterior cranial fossa tumors demonstrated a decrease in CSF infection rate with the use of antibiotic-impregnated catheters compared to the rate in their historical control group..$^{14}$ In an attempt to decrease EVD-associated infection, our pediatric neurosurgical team has exclusively used antibiotic-impregnated EVD catheters since 2011. This study tests the hypothesis that the use of antibioticimpregnated EVD catheters in our pediatric neurosurgical patients demonstrates a decrease in the EVD-associated infection rate compared to our previous study with nonantibiotic-impregnated EVD catheters. ${ }^{15}$

\section{Methods}

We conducted a retrospective cohort observational study in pediatric patients (younger than 18 years of age) in whom antibiotic-impregnated EVDs were placed in the operating room (OR) at The Children's Hospital of Philadelphia between January 1, 2011, and January 1, 2019. The study was approved by the hospital's institutional review board, and the requirement for informed consent was waived.

Patients were identified by the CPT (Current Procedural Terminology) code for burr hole for ventriculostomy placement. They were included if they were treated with a Codman Bactiseal rifampin and clindamycin-coated EVD catheter. Patients were excluded if the EVD was placed through a craniotomy in the OR. For infectionrelated practices, our pediatric neurosurgical team has implemented the following protocol: a sign is placed on the OR door to minimize traffic, the patient is positioned so that the operative site is away from the door, antibiotics are administered prior to incision, hair is clipped and not shaved, ChloraPrep is applied by the attending physician and allowed to dry for 3 minutes, proper hand-washing and double-gloving technique is used by all team members, ioban is applied to the surgical field, and there is one incision site for antibiotic-impregnated EVD placement. Postoperatively, all patients are admitted to our ICU. All of the patients receive perioperative intravenously administered cefazolin or vancomycin (for cephalosporin allergy) for 24 hours. Only the neurosurgical personnel can access the EVD to obtain CSF studies. Indications for CSF cultures to be sent for analysis are fever, wound site dehiscence, or CSF leakage.

For patients with documented positive CSF Gram stain or infections, the antibiotics were vancomycin and cefepime or vancomycin and ciprofloxacin (for cephalosporin allergy) for at least 24 hours, and CSF sampling for infection was performed by the pediatric neurosurgical team every 24-48 hours for at least 3 additional samples. Study data were collected and managed using REDCap electronic data capture tools hosted at The Children's Hospital of Philadelphia. ${ }^{2}$ Variables collected included demographics (age, sex, race, and weight); ventriculostomy indication; number of days ventriculostomy was present; CSF culture findings; ventriculostomy disposition (removed or shunt); and survival from hospital admission to discharge. On par with our prior study, CSF cultures were only sent for patients in whom there was concern for infection and prior to administration of antibiotics. Furthermore, positive CSF cultures obtained at the time of EVD placement or externalization of a ventriculoperitoneal shunt were not included in the overall infection rate. Subsequent positive cultures from patients with previous ventriculoperitoneal shunt infections were included in the overall infection rate only if the CSF cultures were initially negative or if the organism identified on subsequent cultures was different from the initial culture. Daily CSF output values were recorded for up to the first 30 days of EVD management.

\section{Statistical Analysis}

Statistical analysis was performed using SAS software, version 9.4 (SAS Institute). The median and interquartile ranges (IQRs) were presented for continuous variables, whereas frequency and percentages were presented for categorical variables. To compare patients' characteristics and CSF culture outcomes by neurological diagnosis, Kruskal-Wallis tests were used for continuous variables, whereas chi-square or Fisher's exact tests, as appropriate, were used for categorical variables. If the omnibus test was significant, post hoc pairwise analysis was used to explore which diagnosis was different from others. A Bonferroni correction was used to account for multiple comparisons for post hoc analysis $(5 * 4 / 2=10$ in total), with the corrected critical $\mathrm{p}$ value being $0.05 / 10=0.005$. A z-test for differences in two proportions was used to assess the difference in positive culture rate between the current study and the previously published study (2005-2009). ${ }^{15}$

\section{Results}

Two hundred twenty-nine patients with antibiotic-impregnated EVD catheters were included in the analysis, and of this cohort 2 patients $(0.9 \%$; $95 \%$ CI $0 \%, 2.1 \%)$ had an EVD-associated infection highlighted-see cases 1 and 2 below. There were 5 main neurological diagnostic groups for EVD placement: these included externalization of an existing single antibiotic-impregnated shunt (externalized shunt) in 34 patients (14.9\%); brain tumor (tumor) in 77 patients (33.6\%); intracranial hemorrhage (ICH) in 27 patients (11.8\%); traumatic brain injury (TBI) in 6 patients (2.6\%); and 85 patients (37.1\%) were captured in an "other" category (Table 1). The "other" category included patients with pseudomeningocele and CSF leak after CNS surgery and patients with EVD placement at the time of myelomeningocele closure.

There was a difference in age among the neurological diagnostic groups. Patients with "other" diagnoses were statistically younger than patients diagnosed with exter- 
TABLE 1. Association of variable with neurological diagnostic group

\begin{tabular}{|c|c|c|c|c|c|c|c|}
\hline Variable & Total & Ext Shunt & Tumor & $\mathrm{ICH}$ & $\mathrm{TBI}$ & Other & $\mathrm{p}$ Value \\
\hline No. of patients & 229 & $34(14.9 \%)$ & $77(33.6 \%)$ & $27(11.8 \%)$ & $6(2.6 \%)$ & $85(37.1 \%)$ & \\
\hline Age in yrs & $3.4(0.9-9.4)$ & $3.9(2.2-11.2)$ & $4.31(1.1-9.7)$ & $8.6(0.5-13.5)$ & $5.4(3.3-10.0)$ & $1.3(0.1-5.1)$ & $<0.001$ \\
\hline Sex: male & $144(62.9 \%)$ & $19(55.9 \%)$ & $48(62.3 \%)$ & $17(63.0 \%)$ & $3(50.0 \%)$ & $57(67.1 \%)$ & 0.7505 \\
\hline Sex: female & $85(37.1 \%)$ & $15(44.1 \%)$ & $29(37.7 \%)$ & $10(37.0 \%)$ & $3(50.0 \%)$ & $28(32.9 \%)$ & \\
\hline Weight in $\mathrm{kg}$ & $15(9.3-32.7)$ & $16(10.3-35.5)$ & $18(10.5-33.6)$ & $25.9(6.6-49)$ & $23.1(15-32.3)$ & $12.4(3.3-19.5)$ & 0.0012 \\
\hline Max daily EVD output in mL & $248(107-347)$ & $230.5(119-289)$ & $264(153-376)$ & $265.5(124.5-337)$ & $238(195-411)$ & $225(78-415)$ & 0.5384 \\
\hline EVD duration in days & $6(3-10)$ & $5(3-8)$ & $4(2-8)$ & $7(3-12)$ & $9.5(6-14)$ & $7(4-12)$ & 0.0044 \\
\hline Mortality & $29(12.7 \%)$ & $2(5.9 \%)$ & $12(15.6 \%)$ & $4(14.8 \%)$ & $0(0)$ & $11(12.9 \%)$ & 0.6557 \\
\hline
\end{tabular}

Ext $=$ externalized.

Values in parentheses represent either the range or the $\%$, as indicated.

nalized shunt, tumor, and ICH (Table 1). Patients' weight was also statistically less in the "other" diagnosis than in those who were diagnosed with tumor (Table 1).

One hundred ninety-seven patients had EVD drainage for more than 24 hours (not clamped), and the maximum daily EVD output was not significantly different between the groups. The median maximum daily outputs (in milliliters) among all the groups were very similar (range 225-265.5 mL). There was also no significant difference in mortality (Table 1).

The EVD duration was significantly different among groups (Table 1); patients with an EVD placed for intracranial tumor had a significantly shorter EVD duration (median [IQR]: 4 [2-8] days) than those in the "other" group (median [IQR]: 7 [4-12]) days) $(\mathrm{p}=0.0009)$.

Of the total of 229 patients, $100(43 \%)$ had CSF cultures sent on EVD insertion. Patients with externalized shunts due to malfunction, the tumor group, and the "other" group were the majority of those who had a CSF culture sent due to fever, CSF leak, or wound dehiscence, which accounted for 84 of the 100 patients.

We report an EVD-associated infection rate of $0.9 \%$, or 0.99 per 1000 catheter (EVD) days. Characteristics by infection group are presented in Table 2 . There were only 2 patients with positive cultures, so statistical analysis was not done between the infection and no-infection groups because $\mathrm{p}$ values would be uninformative (Table 3 ).

In comparison to our previously published cohort, the infection rate with antibiotic-impregnated EVDs is significantly lower than nonantibiotic-impregnated EVDs $(0.9 \%$ vs $6 \%, p=0.00128) .{ }^{15}$

TABLE 2. Characteristics associated with infection

\begin{tabular}{lcc}
\hline \multicolumn{1}{c}{ Characteristic } & No Positive Culture & Positive Culture \\
\hline No. of patients & $227(99.13 \%)$ & $2(0.87 \%)$ \\
\hline Age in yrs & $3.40(0.88-9.44)$ & $8.38(0.53-16.24)$ \\
\hline Max EVD output in $\mathrm{mL}$ & $246(103-347)$ & $321(295-347)$ \\
\hline Shunt placement & $108(47.58 \%)$ & $0(0)$ \\
\hline Mortality & $29(12.78 \%)$ & $0(0)$ \\
\hline EVD duration in days & $6(3-10)$ & $15.5(13-18)$ \\
\hline
\end{tabular}

Values in parentheses represent either the range or the $\%$, as indicated.

\section{Case 1}

A 6-month-old girl with a history of subarachnoid hemorrhage and intraventricular hemorrhage from a ruptured middle cerebral artery aneurysm underwent placement of an antibiotic-impregnated EVD on hospital day 0. On hospital day 1, she underwent a craniotomy for aneurysm clipping and the same EVD was in place through hospital day 14. She developed a fever on hospital day 14 and CSF cultures that were sent for analysis were positive for non-anthracis Bacillus species. Three subsequent cultures were negative by 6 days. Her EVD was removed, and she did not require a permanent shunt.

\section{Case 2}

A 16-year-old boy who had an intraventricular meningioma resection and who had an antibiotic-impregnated EVD placed on hospital day 0 developed fevers on hospital day 12, and CSF culture was positive for Enterobacter aerogenes. He had the EVD removed and a new antibioticimpregnated EVD was placed. Four days after EVD replacement and after 2 sets of negative cultures, he had another positive culture for E. aerogenes. Three subsequent cultures were negative by 7 days. His EVD was removed, and he did not require a permanent shunt.

Of the 24 infected patients in the initial published cohort who were initially treated using nonantibiotic-impregnated EVD catheters, ${ }^{15} 20$ patients eventually cleared the infection by 1 week (documented by 3 additional negative cultures done every 24 to 48 hours); the other 4 infected patients needed removal of the initial EVD and placement of a new nonantibiotic-impregnated EVD, and they required a total of 2 weeks to clear the infection (doc-

TABLE 3. Comparison of infection rate in this study to a previous study

\begin{tabular}{lccc}
\hline \multicolumn{1}{c}{ Variable } & Test & z Score & p Value \\
\hline $\begin{array}{l}\text { Two-proportion z-test (rate), present } \\
\text { study }\end{array}$ & 2-tailed & -3.218 & 0.00128 \\
\hline $\begin{array}{l}\text { Two-proportion z-test (per 1000 } \\
\quad \text { catheter days), previous study by }\end{array}$ & 2-tailed & -3.5451 & 0.00038 \\
$\quad$ Topjian et al. & & & \\
\hline
\end{tabular}


umented by 3 additional negative cultures done every 24 to 48 hours). Although cell count trends can be helpful, we primarily rely on 3 successive cultures to document clearance of infection.

\section{Discussion}

Of the 229 pediatric patients who underwent a burr hole for antibiotic-impregnated EVD placement over an 8 -year period, our EVD-associated infections were very rare, with only 2 patients $(0.9 \%)$ having a positive CSF culture. This results in an infection rate of 0.99 per 1000 catheter days. In our previous study, in which we used nonantibiotic-impregnated EVD catheters in pediatric patients, the infection rate was higher at 6\% (8.6 per 1000 catheter days).${ }^{15}$ These data demonstrate that there was a decrease in EVD-associated CSF infections while using antibiotic-impregnated catheters compared to a historical control group of pediatric patients with nonantibiotic-impregnated EVD catheters.

In the hopes of decreasing our CSF infection rate, our pediatric neurosurgery group has exclusively used the antibiotic-impregnated EVD catheters since 2011, which was the main change in the care of the patients between our prior published cohort and the current cohort. Although we did see a decrease in the infection rate in the current study compared to our previous study in which nonantibiotic-impregnated EVD catheters were used, the total number of patients in the current study was smaller than the total number of patients in the previous study (229 vs 380, respectively). ${ }^{15}$ The underlying reasons for EVD placement in the patients were also slightly different; whereas the majority of the patients in the current study were from the "other" group and the "tumor" group, the "externalized shunt" and "tumor" groups predominated in the previous study. The patients with the "other" diagnosis in the current study were younger and thus had a lower weight than patients diagnosed with externalized shunt, tumor, and ICH. This is due to the fact that the majority of the patients in the "other" category underwent placement of antibiotic-impregnated EVDs for postnatal myelomeningocele closure (neonate). The remaining population of patients in the "other" category were patients with CSF leakage or pseudomeningocele, which is more common in neonates and infants because of their thinner skin.

In the current study, patients diagnosed with "externalized shunt" were more likely than those diagnosed with "tumor" to have a CSF culture sent for analysis on EVD insertion. This is probably because patients whose existing shunts need to be externalized may have a concern for infection in the first place, leading to externalization. Also, patients who have externalized shunts may need to show negative cultures prior to shunt revision clearance. This is consistent with our previous study in which the majority of the patients diagnosed with "externalized shunt" had CSF cultures sent on EVD insertion. ${ }^{15}$

Previously, we reported that pediatric patients with TBI and meningitis had the highest incidence of EVDassociated infection. ${ }^{15}$ In contrast, our 2 infection cases in the current study were in neither category; 1 was an intraventricular hemorrhage from an aneurysm and 1 was an intraventricular meningioma. We also previously reported longer EVD durations and higher maximum EVD output as a risk factor for infection; however, in the current study, because there were only 2 cases of EVD-associated infection, we could not determine statistically significant risk factors associated with EVD infection. Both of the cases of infection in the current study, however, had positive cultures for CSF infection 12 days or more after antibiotic-impregnated EVD placement. In our previous study, the majority of infections were observed in the first 10 days after nonantibiotic-impregnated EVD catheter placement.

Recently there has been an increase in the number of studies on placement of antibiotic-impregnated catheters in adult patients, with most but not all studies demonstrating a reduction in EVD-associated infections. ${ }^{4,711,13,16} \mathrm{In}$ contrast, studies are very limited in the pediatric literature. In one recent study in which a prospective, multicenter analysis of EVD-related infections in the UK and Ireland were reported, the majority of the patients were adults in whom the type of catheter had no effect on the adult infection rate; of the 37 pediatric patients in the study, 3 had an infection (an infection rate of 8.1\%), and 23 of the 37 pediatric patients had an antibiotic-impregnated catheter, but no information is given about whether the infection was associated with the type of catheter (antibiotic-impregnated, silver-impregnated, or nonantibiotic-impregnated). ${ }^{4}$ In a multicenter pediatric study involving 1670 patients in North America, the use of antibiotic-impregnated catheters for shunt procedures did not reduce the infection rate compared to historical controls of patients who were treated with nonantibiotic-impregnated catheters-but the patients who needed EVDs were excluded from this study. ${ }^{5}$ In a single-institution retrospective cohort pediatric study of patients with posterior fossa tumors with hydrocephalus, the CSF infection rate of $2.1 \%$ in the 47 patients who were managed with antibiotic-impregnated EVD catheters was significantly lower than the infection rate of $31.8 \%$ in their historical control group of 44 patients who were managed with nonantibiotic-impregnated EVD catheters. ${ }^{14}$ Our data add a significant number of pediatric patients to the literature on antibiotic-impregnated EVDs in the pediatric population and show a significant decrease in the number of EVD-associated infections since the change to antibiotic-impregnated catheters at our institution.

\section{Study Limitations}

There are several limitations to our study that must be considered when attempting to generalize our findings. This is a retrospective study of only 4 pediatric neurosurgeons' operative practices in a single institution, which may not be applicable to most institutions. All of our pediatric neurosurgical patients are admitted to our ICU for the duration of their EVD management, undergoing daily rounds by our pediatric critical care team and daily discussions with our pediatric neurosurgical team, which may have contributed to the relatively low infection rate. The distribution of patients by neurological diagnostic group was heavily weighted to younger patients with open myelomeningoceles or CSF leaks/pseudomeningoceles. Even though none of these patients had an EVD- 
associated infection, in many of them the EVD was converted to a shunt after a number of days. Any subsequent shunt infections after EVD removal were not analyzed because it was assumed that any infection was a result of the shunt placement and not the original EVD placement. Therefore, our experience may not be representative of infection rates at other pediatric or combined pediatric/ adult institutions that may not have similar tumor, shunt, and open myelomeningocele populations. In addition, our sample size is small in the TBI category because we typically do not see a large number of patients with TBI at our institution, and this population is usually not managed with EVDs. We also did not analyze the number of bedside EVD placements, because all EVDs analyzed in this study were placed in the OR under a more sterile condition. Furthermore, we did not prospectively compare the infection rate in patients managed with antibiotic-impregnated EVD catheters versus patients managed with nonantibiotic-impregnated EVD catheters during the same time period.

\section{Future Directions}

Further studies are needed at other institutions to corroborate or disprove our findings. If other institutions corroborate our findings, a multicenter prospective study in pediatric patients comparing the infection rate of antibiotic-impregnated EVD catheters versus nonantibioticimpregnated EVD catheters during the same time period is warranted. Furthermore, another study can compare the subsequent incidence of internalized shunt infections following the use of antibiotic-impregnated versus nonantibiotic-impregnated EVDs. Whether antibiotic-impregnated EVD catheters truly decrease the rate of infection or just delay the onset of infection and whether they promote different or resistant infections are other areas for future research.

\section{Conclusions}

To the best of our knowledge, this study represents the largest cohort of pediatric patients focusing on risk factors associated with CSF infection from EVD placement. Since the implementation of antibiotic-impregnated EVDs at our institution, positive CSF cultures during EVD management have been extremely rare and significantly lower than in our prior cohort treated with nonantibiotic-impregnated EVDs.

\section{References}

1. Bell MJ, Carpenter J, Au AK, Keating RF, Myseros JS, Yaun A, et al: Development of a pediatric neurocritical care service. Neurocrit Care 10:4-10, 2009

2. Harris PA, Taylor R, Thielke R, Payne J, Gonzalez N, Conde JG: Research electronic data capture (REDCap) - a metadata-driven methodology and workflow process for providing translational research informatics support. J Biomed Inform 42:377-381, 2009

3. Ifeachor AP, Nichols KR, Morris JL, Cox EG, Smith JL, Sinclair EA: Antimicrobial prophylaxis for external ventricular drains in pediatric neurosurgical patients. Paediatr Drugs 17:239-244, 2015

4. Jamjoom AAB, Joannides AJ, Poon MT, Chari A, Zaben
M, Abdulla MAH, et al: Prospective, multicentre study of external ventricular drainage-related infections in the UK and Ireland. J Neurol Neurosurg Psychiatry 89:120-126, 2018

5. Kestle JR, Holubkov R, Cochrane D, Kulkarni AV, Limbrick DD Jr, Luerssen TG, et al: A new Hydrocephalus Clinical Research Network protocol to reduce cerebrospinal fluid shunt infection. J Neurosurg Pediatr 17:391-396, 2016

6. LaRovere KL, Graham RJ, Tasker RC: Pediatric neurocritical care: a neurology consultation model and implication for education and training. Pediatr Neurol 48:206-211, 2013

7. Mikhaylov Y, Wilson TJ, Rajajee V, Thompson BG, Maher CO, Sullivan SE, et al: Efficacy of antibiotic-impregnated external ventricular drains in reducing ventriculostomyassociated infections. J Clin Neurosci 21:765-768, 2014

8. Muttaiyah S, Ritchie S, John S, Mee E, Roberts S: Efficacy of antibiotic-impregnated external ventricular drain catheters. J Clin Neurosci 17:296-298, 2010

9. Root BK, Barrena BG, Mackenzie TA, Bauer DF: Antibiotic impregnated external ventricular drains: meta and cost analysis. World Neurosurg 86:306-315, 2016

10. Shekhar H, Kalsi P, Dambatta S, Strachan R: Do antibioticimpregnated external ventriculostomy catheters have a low infection rate in clinical practice? A retrospective cohort study. Br J Neurosurg 30:64-69, 2016

11. Sheppard JP, Ong V, Lagman C, Udawatta M, Duong C, Nguyen T, et al: Systemic antimicrobial prophylaxis and antimicrobial-coated external ventricular drain catheters for preventing ventriculostomy-related infections: a metaanalysis of 5242 cases. Neurosurgery [epub ahead of print], 2018

12. Sonabend AM, Korenfeld Y, Crisman C, Badjatia N, Mayer SA, Connolly ES Jr: Prevention of ventriculostomy-related infections with prophylactic antibiotics and antibioticcoated external ventricular drains: a systematic review. Neurosurgery 68:996-1005, 2011

13. Talibi S, Tarnaris A, Shaw SA: Has the introduction of antibiotic-impregnated external ventricular drain catheters changed the nature of the microorganisms cultured in patients with drain-related infection? A single neurosurgical centre's experience. Br J Neurosurg 30:560-566, 2016

14. Tamburrini G, Massimi L, Caldarelli M, Di Rocco C: Antibiotic impregnated external ventricular drainage and third ventriculostomy in the management of hydrocephalus associated with posterior cranial fossa tumours. Acta Neurochir (Wien) 150:1049-1056, 2008

15. Topjian AA, Stuart A, Pabalan AA, Clair A, Kilbaugh TJ, Abend NS, et al: Risk factors associated with infections and need for permanent cerebrospinal fluid diversion in pediatric intensive care patients with externalized ventricular drains. Neurocrit Care 21:294-299, 2014

16. Verberk JDM, Berkelbach van der Sprenkel JW, Arts MP, Dennesen PJ, Bonten MJ, van Mourik MSM: Preventing ventriculostomy-related infections with antibioticimpregnated drains in hospitals: a two-centre Dutch study. J Hosp Infect 92:401-404, 2016

17. Wright K, Young P, Brickman C, Sam T, Badjatia N, Pereira $\mathrm{M}$, et al: Rates and determinants of ventriculostomy-related infections during a hospital transition to use of antibioticcoated external ventricular drains. Neurosurg Focus 34(5):E12, 2013

\section{Disclosures}

The authors report no conflict of interest concerning the materials or methods used in this study or the findings specified in this paper. 
Lang et al.

\section{Author Contributions}

Conception and design: Lang, Huh. Acquisition of data: Lang, Yver, Palma, Huh. Analysis and interpretation of data: Lang, Zhang, Huh. Drafting the article: Lang, Zhang, Huh. Critically revising the article: Lang, Zhang, Kirschen, Topjian, Kennedy, Storm, Heuer, Mensinger, Huh. Reviewed submitted version of manuscript: Lang, Zhang, Palma, Kirschen, Topjian, Kennedy, Storm, Heuer, Mensinger, Huh. Approved the final version of the manuscript on behalf of all authors: Lang. Statistical analysis: Zhang, Mensinger. Administrative/technical/material support: Lang, Huh. Study supervision: Lang, Huh.

\section{Correspondence}

Shih-Shan Lang: Children's Hospital of Philadelphia, PA. chens4@email.chop.edu. 